BMC

Pediatrics

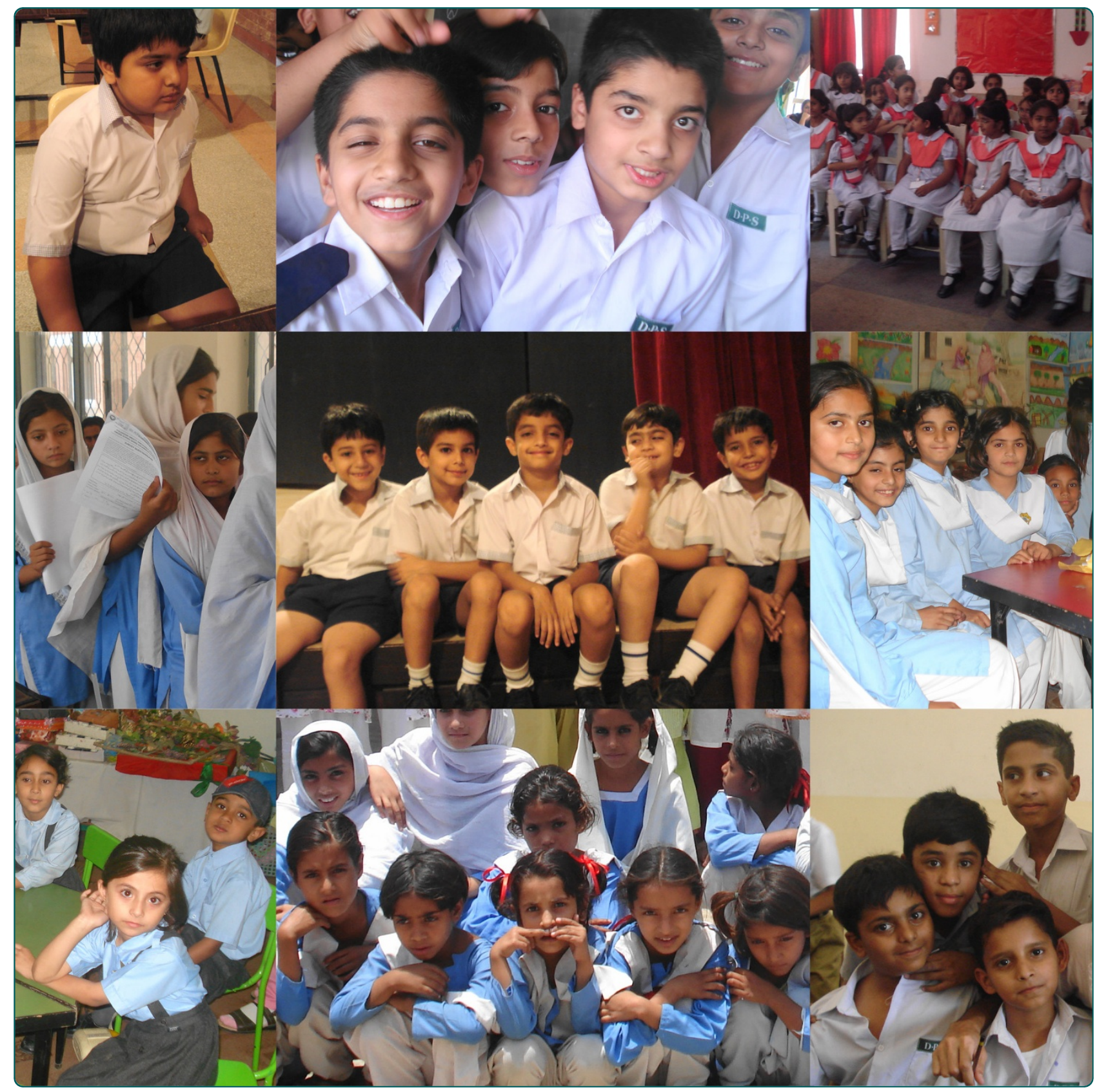

Waist circumference, waist-hip ratio and waistheight ratio percentiles and central obesity among Pakistani children aged five to twelve years

Mushtaq et al. 


\title{
Waist circumference, waist-hip ratio and waist- height ratio percentiles and central obesity among Pakistani children aged five to twelve years
}

\author{
Muhammad Umair Mushtaq ${ }^{1,2^{*}}$, Sibgha Gull', Hussain Muhammad Abdullah', Ubeera Shahid ${ }^{1}$,
} Mushtaq Ahmad Shad ${ }^{2}$ and Javed Akram

\begin{abstract}
Background: Central obesity has been associated with the risk of cardiovascular and metabolic disease in children and anthropometric indices predictive of central obesity include waist circumference (WC), waist-hip ratio (WHR) and waist-height ratio (WHtR). South Asian children have higher body fat distribution in the trunk region but the literature regarding WC and related indices is scarce in this region. The study was aimed to provide age- and gender-specific WC, WHR and WHtR smoothed percentiles, and to explore prevalence and correlates of central obesity, among Pakistani children aged five to twelve years.
\end{abstract}

Methods: A population-based cross-sectional study was conducted with a representative multistage random cluster sample of 1860 primary school children aged five to twelve years in Lahore, Pakistan. Smoothed percentile curves were constructed for WC, WHR and WHtR by the LMS method. Central obesity was defined as having both age- and gender-specific WC percentile $\geq 90^{\text {th }}$ and WHtR $\geq 0.5$. Chi-square test was used as the test of trend. Multivariate logistic regression was used to quantify the independent predictors of central obesity and adjusted odds ratios (aOR) with 95\% Cl were obtained. Linear regression was used to explore the independent determinants of WC and WHtR. Statistical significance was considered at $P<0.05$.

Results: First ever age- and gender-specific smoothed WC, WHR and WHtR reference curves for Pakistani children aged five to twelve years are presented. WC increased with age among both boys and girls. Fiftieth WC percentile curves for Pakistani children were higher as compared to those for Hong Kong and British children, and were lower as compared to those for Iranian, German and Swiss children. WHR showed a plateau pattern among boys while plateau among girls until nine years of age and decreased afterwards. WHtR was age-independent among both boys and girls, and WHtR cut-off of $\geq 0.5$ for defining central obesity corresponded to $85^{\text {th }}$ WHtR percentile irrespective of age and gender. Twelve percent children $(95 \% \mathrm{Cl} 10.1-13.0)$ had a WC $\geq 90^{\text {th }}$ percentile and $16.5 \%$ children $(95 \% \mathrm{Cl} 14.7-18.1)$ had a WHtR $\geq 0.5$ while $11 \%$ children $(95 \% \mathrm{Cl} 8.9-11.6)$ had both $\mathrm{WC} \geq 90^{\text {th }}$ percentile and WHtR $\geq 0.5$. Significant predictors of central obesity included higher grade, urban area with high socioeconomic status (SES), high-income neighborhood and higher parental education. Children studying in higher grade (aOR 5.11, 95\% Cl 1.76-14.85) and those living in urban area with high SES (aOR 82.34, 95\% Cl 15.76-430.31) showed a significant independent association. Urban area with high SES and higher parental education showed a significant independent association with higher WC and higher WHtR while higher grade showed a significant independent association with higher WC.

Conclusions: Comprehensive worldwide reference values are needed to define central obesity and the present study is the first one to report anthropometric indices predictive of central obesity for Pakistani school-aged children. Eleven percent children were centrally obese and strong predictors included higher grade, urban area

\footnotetext{
* Correspondence: mushtaqmu@gmail.com

'Ubeera Memorial Research Society, Allama Iqbal Medical College, Lahore 54000, Pakistan

Full list of author information is available at the end of the article
}

\section{Biomed Central}

(c) 2011 Mushtaq et al; licensee BioMed Central Ltd. This is an Open Access article distributed under the terms of the Creative Commons Attribution License (http://creativecommons.org/licenses/by/2.0), which permits unrestricted use, distribution, and reproduction in any medium, provided the original work is properly cited. 
with high SES and higher parental education. These findings support the need for developing a National strategy for childhood obesity and implementing targeted interventions, prioritizing the higher social class and involving communities.

\section{Background}

Obesity is a global epidemic and children are the worst affected with an estimated ten percent of school-aged children being overweight and one quarter of these being obese worldwide [1]. Childhood obesity epidemic is now penetrating the developing countries including Pakistan, especially in the affluent urban population [2,3]. Central obesity has been associated with the risk of cardiovascular and metabolic disease in children and anthropometric indices predictive of childhood central obesity include waist circumference (WC), waist-hip ratio (WHR) and waist-height ratio (WHtR) [4-8]. However, measurement of WC is not yet a common practice in children. Most of the studies reporting percentiles for WC and related indices in school-aged children were conducted in the developed countries including United States, Australia and European region [9-19]. In Asia, WC percentiles have been reported for Middle-eastern and Southeast Asian children [20-24]. South Asian children have higher body fat distribution in the trunk region [25-29]; however, the data on WC and related indices is scarce in this region. Comprehensive worldwide reference values for WC and related indices are needed to establish an internationally accepted age-, gender-, and ethnicity-specific definition of central obesity in children. The study was aimed to provide first ever age- and gender-specific WC, WHR and WHtR smoothed percentiles, and to explore prevalence and correlates of central obesity, among Pakistani children aged five to twelve years.

\section{Methods}

\section{Design, setting and sample}

A population-based cross-sectional study, the Nutritional Assessment among School-going Children in Lahore, Pakistan (NASCL study), was conducted among primary school children aged five to twelve years in 2009-10. Lahore is the capital of Pakistan's most populous province Punjab and a metropolis with multiethnic populations. It has a population of nine million, including 2.5 million primary school children, and $81 \%$ of the population resides in urban area (Administrative data, Government of Punjab, 2010).

A stratified multistage random cluster sample of 1860 children aged five to twelve years in twelve primary schools of City District Lahore was enrolled. Stratified sampling, based on the population and educational system characteristics, was used to have proportionate representation of gender, area of residence and socioeconomic status
(SES). About 1200 public and 1100 private primary schools in Lahore registered with the Punjab Department of Education were listed. The listed schools were stratified according to the geographic area and monthly fee structure of schools into following four strata: a) urban with high SES (urban area and fee $>2500$ PKR), b) urban with middle SES (urban area and fee $=1000-2500$ PKR), c) urban with low SES (urban area and fee $<1000$ PKR), and d) rural with low/disadvantaged SES (rural area and fee $\sim 100$ PKR or free). The former two strata included private (including public-private mix) schools and the later two strata included public schools. In Pakistan, public schools cater low SES urban and rural children while high and middle SES urban children are educated in private and public-private mix schools. Three schools were selected at random from each stratum and contacted by the Departments of Education and Health to participate voluntarily in the study. If school administration refused to participate, next school was selected randomly from the respective stratum. For each school, a list of all classes in five grades (one to five) was obtained and one class in each grade was selected at random. In this way, sixty classes, five from each school, were selected. For each of the selected classes, first thirty-one children on class attendance register, present on data collection day and aged five to twelve years, were included in the study. Children suffering from any known metabolic syndrome (like Prader-Willi syndrome) and those not willing to participate in the study were excluded. Sample size was calculated using Epi Info 6.04d (United States' Centers for Disease Control and Prevention, 2004) with a confidence (1- $\alpha)$ of $95 \%$, anticipated prevalence of $5 \%$ and margin of error of \pm 1 . The minimum sample size calculated was 1823 and a sample of 1860 was deemed sufficient.

\section{Data Collection}

The sampled schools were visited on pre-arranged dates in summer 2009 by a team of trained senior medical students lead by the Principal Investigator. Health education of children and teachers was also carried out after data collection in the respective school. Height was measured by portable stadiometers that were standardized and calibrated before the measurements. Feet were placed together with heels, buttocks and shoulder blades against the stick and head was positioned in the Frankfurt horizontal plane. WC and hip circumference $(\mathrm{HC})$ were measured according to the World Health Organization (WHO) recommendations [30,31]. The subject was asked 
to stand relaxed with arms at the sides, feet positioned close together and weight evenly distributed across feet. WC was measured midway between the lowest rib and the superior border of iliac crest at the end of normal expiration with a stretch-resistant measuring tape positioned at a level parallel to the floor. $\mathrm{HC}$ was measured at the level of widest portion of buttocks (trochanters). All measurements were in centimeters $(\mathrm{cm})$ to the nearest $0.1 \mathrm{~cm}$. All children were measured in mornings or early afternoons without shoes and in light summer schooluniform.

Demographic information of all officially enrolled students in the sampled classes was obtained before data collection that included gender, date of birth, residential address and parental education. If demographic information of students was not found on official rosters, it was obtained from the classroom teachers. Parental education level was based on the parent with the highest total years of schooling and neighborhood income level was based on the approximate income estimate of child's residential area obtained from the Revenue Department of City District Government Lahore. Quality control measures and good practices including training, pre-testing the processes and materials, field monitoring of data collection, logistics management and daily meetings of the study teams were ensured. Informed consent statement was printed on the study form. Verbal informed consent for the child to participate in the study was taken from class teachers and school heads. As the study involved no invasive procedure, verbal informed consent was deemed sufficient. The study was approved by the Ethical Review Board of Allama Iqbal Medical College, Lahore. Permissions to conduct the survey were granted by the Punjab Departments of Education and Health, and the sampled schools.

\section{Statistical Analysis}

Data were entered and analyzed by manual and computerized checking using SPSS version 18.0 (SPSS Inc. Chicago IL, United States, 2009). Age was calculated to the precise day by subtracting the date of birth from the date of examination. Smoothed age- and gender-specific percentile curves were constructed for WC, WHR and WHtR by the LMS method [32]. Fiftieth percentile curves for WC were compared with the previous studies in Hong Kong, United Kingdom, Iran, Germany, Switzerland and China that measured WC at the same site. Cut-off of $\geq 90^{\text {th }}$ age- and gender-specific percentile for WC $[15,17,18,24,33,34]$ and $\geq 0.5$ for WHtR [15,35-37] had been suggested for defining central obesity. Central obesity was defined as having both age- and gender-specific WC percentile $\geq 90^{\text {th }}$ and WHtR $\geq 0.5$. Bivariate analysis, using chi-square test as the test of trend, was conducted to compare the prevalence of central obesity among the study variables. Multivariate logistic regression was used to quantify the independent predictors of central obesity and adjusted odds ratios (aOR) with 95\% confidence interval (CI) were obtained. Linear regression was used to explore the predictive power of all socio-demographic factors significantly associated with central obesity (as independent variables) in relation to WC and WHtR (as dependent variables). Statistical significance was considered at $\mathrm{P}<0.05$ and all tests were two-sided.

\section{Results}

The study included a sample of 1860 primary school children aged five to twelve years. The male-female ratio was 1.11 with $52.5 \%$ boys and $47.5 \%$ girls. The sample involved 20\% children from each grade and $25 \%$ children from each area and SES stratum. Seventy-five percent children were urban and $25 \%$ were rural. The median age (range) was 101.2 (60-154) months. The mean and standard deviation (SD) for WC, HC, height, WHR and WHtR were $58.4(7.9) \mathrm{cm}, 67.2(11.4) \mathrm{cm}, 128.4(11.4)$ $\mathrm{cm}, 0.88(0.17)$ and $0.46(0.05)$ respectively. Age- and gender-specific mean (SD) WC, WHR and WHtR are presented in Table 1.

Age- and gender-specific WC percentiles [Table 2] [Figure 1], age- and gender-specific WHR percentiles [Table 3] [Figure 2], and age- and gender-specific WHtR percentiles [Table 4] [Figure 3] were developed and smoothed by the LMS method. WC increased with age among both boys and girls. Fiftieth WC percentile curves for Pakistani children were higher as compared to those

\begin{tabular}{|c|c|c|c|c|}
\hline & $\mathrm{n}$ & $W C(\mathrm{~cm})$ & WHR & WHtR \\
\hline \multicolumn{5}{|l|}{ Boys $(n=977)$} \\
\hline 5 years (61-71 months) & 84 & $54.0(5.8)$ & $0.89(0.04)$ & $0.46(0.04)$ \\
\hline 6 years (72-83 months) & 161 & $54.0(5.6)$ & $0.89(0.04)$ & $0.46(0.04)$ \\
\hline 7 years (84-95 months) & 160 & $55.8(5.8)$ & $0.88(0.04)$ & $0.45(0.05)$ \\
\hline 8 years (96-107 months) & 158 & $58.3(6.6)$ & $0.88(0.04)$ & $0.45(0.04)$ \\
\hline 9 years (108-119 months) & 161 & $60.8(7.9)$ & $0.88(0.05)$ & $0.45(0.05)$ \\
\hline 10 years (120-131 months) & 147 & $62.7(9.8)$ & $0.88(0.05)$ & $0.45(0.06)$ \\
\hline 11 years (132-143 months) & 69 & $62.0(7.2)$ & $0.88(0.06)$ & $0.45(0.05)$ \\
\hline 12 years ( $144-155$ months) & 37 & $61.2(5.6)$ & $0.87(0.04)$ & $0.44(0.03)$ \\
\hline \multicolumn{5}{|l|}{ Girls $(n=883)$} \\
\hline 5 years (61-71 months) & 72 & $52.6(3.4)$ & $0.86(0.05)$ & $0.46(0.03)$ \\
\hline 6 years (72-83 months) & 143 & $54.4(6.0)$ & $0.85(0.05)$ & $0.46(0.04)$ \\
\hline 7 years (84-95 months) & 157 & $57.0(7.2)$ & $0.87(0.08)$ & $0.46(0.05)$ \\
\hline 8 years (96-107 months) & 159 & $58.7(7.0)$ & $0.86(0.06)$ & $0.46(0.05)$ \\
\hline 9 years (108-119 months) & 151 & $61.6(8.3)$ & $0.86(0.06)$ & $0.46(0.06)$ \\
\hline 10 years (120-131 months) & 120 & $62.3(8.9)$ & $0.84(0.06$ & $0.45(0.06)$ \\
\hline 11 years (132-143 months) & 62 & $62.3(8.4)$ & $0.80(0.06)$ & $0.43(0.05)$ \\
\hline 12 years (144-155 months) & 19 & $62.6(7.9)$ & $0.81(0.05)$ & $0.43(0.04)$ \\
\hline
\end{tabular}


Table 2 Age- and gender-specific smoothed waist circumference (WC) percentiles for Pakistani primary school children aged five to twelve years ( $\mathrm{n}=1860$ )

\begin{tabular}{|c|c|c|c|c|c|c|c|c|c|}
\hline & \multicolumn{9}{|c|}{ Percentiles } \\
\hline & $3^{\text {rd }}$ & $5^{\text {th }}$ & $10^{\text {th }}$ & $25^{\text {th }}$ & $50^{\text {th }}$ & $75^{\text {th }}$ & $90^{\text {th }}$ & $95^{\text {th }}$ & $97^{\text {th }}$ \\
\hline \multicolumn{10}{|l|}{ Boys $(n=977)$} \\
\hline 5 years (61-71 months) & 45.7 & 46.4 & 47.4 & 49.4 & 52.1 & 55.3 & 59.1 & 61.9 & 64.1 \\
\hline 6 years (72-83 months) & 46.7 & 47.4 & 48.5 & 50.7 & 53.6 & 57.2 & 61.5 & 64.6 & 67.1 \\
\hline 7 years (84-95 months) & 47.7 & 48.5 & 49.7 & 52.1 & 55.3 & 59.4 & 64.1 & 67.7 & 70.5 \\
\hline 8 years (96-107 months) & 48.8 & 49.6 & 51.0 & 53.6 & 57.1 & 61.6 & 66.9 & 70.9 & 74.0 \\
\hline 9 years (108-119 months) & 49.7 & 50.6 & 52.1 & 54.9 & 58.8 & 63.7 & 69.5 & 74.0 & 77.5 \\
\hline 10 years (120-131 months) & 50.5 & 51.4 & 53.0 & 56.1 & 60.3 & 65.6 & 72.0 & 76.9 & 80.7 \\
\hline 11 years (132-143 months) & 51.1 & 52.1 & 53.8 & 57.1 & 61.6 & 67.4 & 74.2 & 79.5 & 83.6 \\
\hline 12 years (144-155 months) & 51.5 & 52.6 & 54.4 & 58.0 & 62.8 & 68.9 & 76.2 & 81.9 & 86.2 \\
\hline \multicolumn{10}{|l|}{ Girls $(n=883)$} \\
\hline 5 years (61-71 months) & 45.8 & 46.4 & 47.4 & 49.3 & 51.8 & 54.7 & 58.0 & 60.4 & 62.1 \\
\hline 6 years (72-83 months) & 46.7 & 47.4 & 48.5 & 50.7 & 53.6 & 57.1 & 61.0 & 63.9 & 66.0 \\
\hline 7 years (84-95 months) & 47.7 & 48.5 & 49.8 & 52.2 & 55.5 & 59.6 & 64.3 & 67.7 & 70.4 \\
\hline 8 years (96-107 months) & 48.7 & 49.6 & 51.0 & 53.8 & 57.5 & 62.2 & 67.6 & 71.8 & 74.9 \\
\hline 9 years (108-119 months) & 49.6 & 50.5 & 52.2 & 55.2 & 59.4 & 64.7 & 70.9 & 75.7 & 79.4 \\
\hline 10 years (120-131 months) & 50.2 & 51.3 & 53.0 & 56.4 & 61.0 & 66.9 & 74.0 & 79.4 & 83.6 \\
\hline 11 years (132-143 months) & 50.6 & 51.8 & 53.7 & 57.4 & 62.5 & 69.0 & 76.8 & 82.8 & 87.4 \\
\hline 12 years (144-155 months) & 50.9 & 52.2 & 54.3 & 58.3 & 63.8 & 70.9 & 79.4 & 85.9 & 91.0 \\
\hline
\end{tabular}



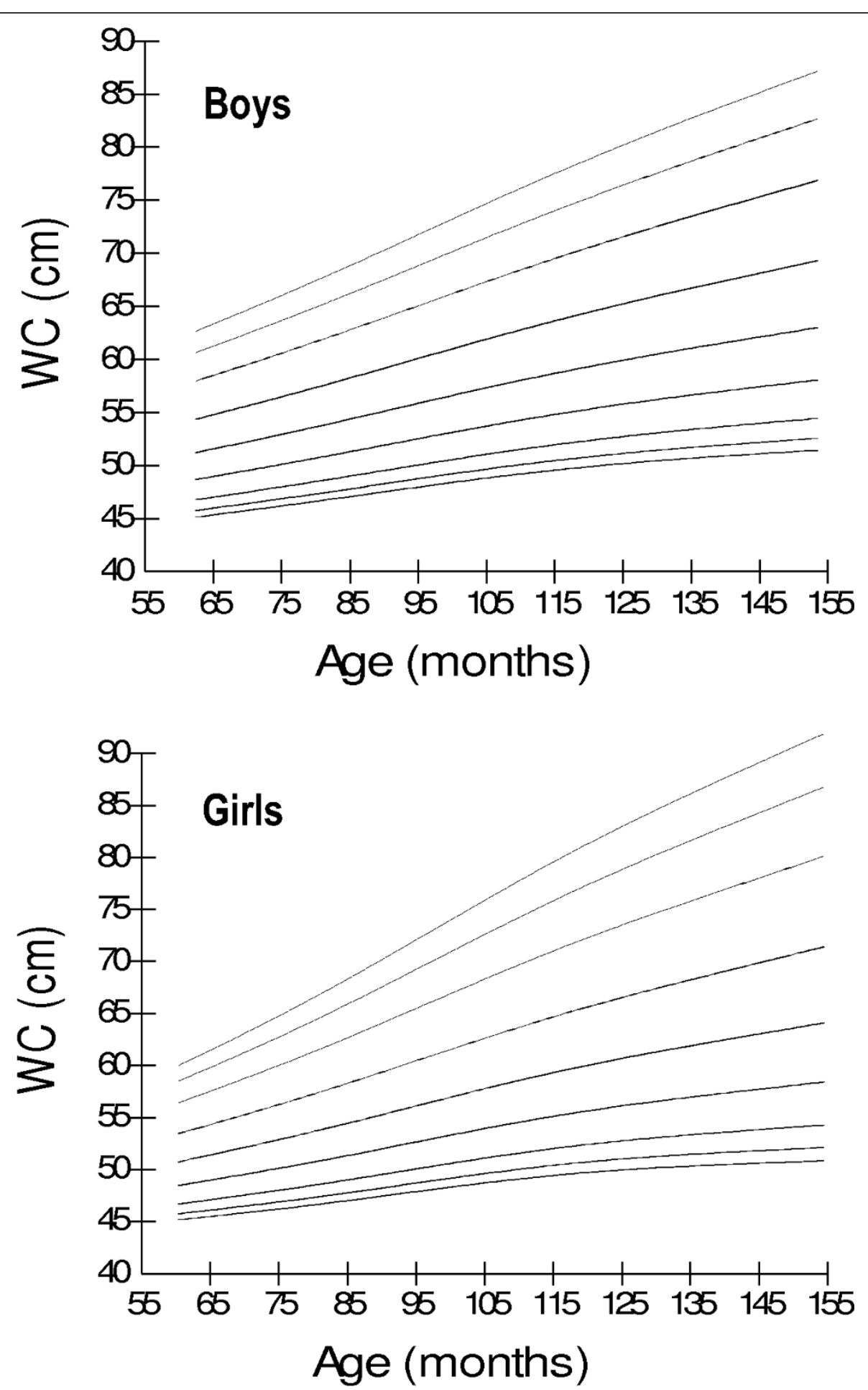

Figure 1 Smoothed waist circumference (WC) percentile curves for Pakistani primary school boys $(n=997)$ and girls $(n=883)$ aged five to twelve years.

for Hong Kong and British children, and were lower as compared to those for Iranian, German and Swiss children [Figure 4]. Fiftieth percentile curves for Chinese children were lower than the study sample in younger age groups and were higher in older children. WHR showed a plateau pattern among boys while plateau among girls until nine years of age and decreased afterwards. WHtR was age-independent among both boys and 
Table 3 Age- and gender-specific smoothed waist-hip ratio (WHR) percentiles for Pakistani primary school children aged five to twelve years ( $\mathrm{n}=1860$ )

\begin{tabular}{|c|c|c|c|c|c|c|c|c|c|}
\hline & \multicolumn{9}{|c|}{ Percentiles } \\
\hline & $3^{\text {rd }}$ & $5^{\text {th }}$ & $10^{\text {th }}$ & $25^{\text {th }}$ & $50^{\text {th }}$ & $75^{\text {th }}$ & $90^{\text {th }}$ & $95^{\text {th }}$ & $97^{\text {th }}$ \\
\hline \multicolumn{10}{|l|}{ Boys $(n=977)$} \\
\hline 5 years (61-71 months) & 0.82 & 0.83 & 0.84 & 0.86 & 0.89 & 0.92 & 0.94 & 0.96 & 0.97 \\
\hline 6 years (72-83 months) & 0.81 & 0.82 & 0.84 & 0.86 & 0.89 & 0.92 & 0.94 & 0.96 & 0.97 \\
\hline 7 years (84-95 months) & 0.80 & 0.81 & 0.83 & 0.86 & 0.88 & 0.91 & 0.94 & 0.96 & 0.97 \\
\hline 8 years (96-107 months) & 0.80 & 0.81 & 0.82 & 0.85 & 0.88 & 0.91 & 0.94 & 0.96 & 0.97 \\
\hline 9 years (108-119 months) & 0.79 & 0.80 & 0.82 & 0.85 & 0.88 & 0.91 & 0.94 & 0.96 & 0.97 \\
\hline 10 years (120-131 months) & 0.78 & 0.79 & 0.81 & 0.84 & 0.88 & 0.91 & 0.94 & 0.96 & 0.97 \\
\hline 11 years (132-143 months) & 0.77 & 0.79 & 0.81 & 0.84 & 0.88 & 0.91 & 0.94 & 0.96 & 0.97 \\
\hline 12 years (144-155 months) & 0.77 & 0.78 & 0.80 & 0.84 & 0.87 & 0.91 & 0.94 & 0.96 & 0.97 \\
\hline \multicolumn{10}{|l|}{ Girls $(n=883)$} \\
\hline 5 years (61-71 months) & 0.76 & 0.77 & 0.79 & 0.82 & 0.85 & 0.89 & 0.93 & 0.95 & 0.97 \\
\hline 6 years (72-83 months) & 0.76 & 0.77 & 0.79 & 0.82 & 0.86 & 0.9 & 0.93 & 0.96 & 0.97 \\
\hline 7 years (84-95 months) & 0.76 & 0.77 & 0.79 & 0.82 & 0.86 & 0.9 & 0.94 & 0.96 & 0.98 \\
\hline 8 years (96-107 months) & 0.75 & 0.76 & 0.78 & 0.82 & 0.86 & 0.9 & 0.94 & 0.97 & 0.98 \\
\hline 9 years (108-119 months) & 0.75 & 0.76 & 0.78 & 0.81 & 0.85 & 0.89 & 0.93 & 0.96 & 0.98 \\
\hline 10 years (120-131 months) & 0.73 & 0.74 & 0.76 & 0.79 & 0.83 & 0.88 & 0.92 & 0.95 & 0.97 \\
\hline 11 years (132-143 months) & 0.72 & 0.73 & 0.74 & 0.78 & 0.82 & 0.86 & 0.90 & 0.93 & 0.95 \\
\hline 12 years (144-155 months) & 0.70 & 0.71 & 0.73 & 0.76 & 0.80 & 0.84 & 0.89 & 0.92 & 0.94 \\
\hline
\end{tabular}



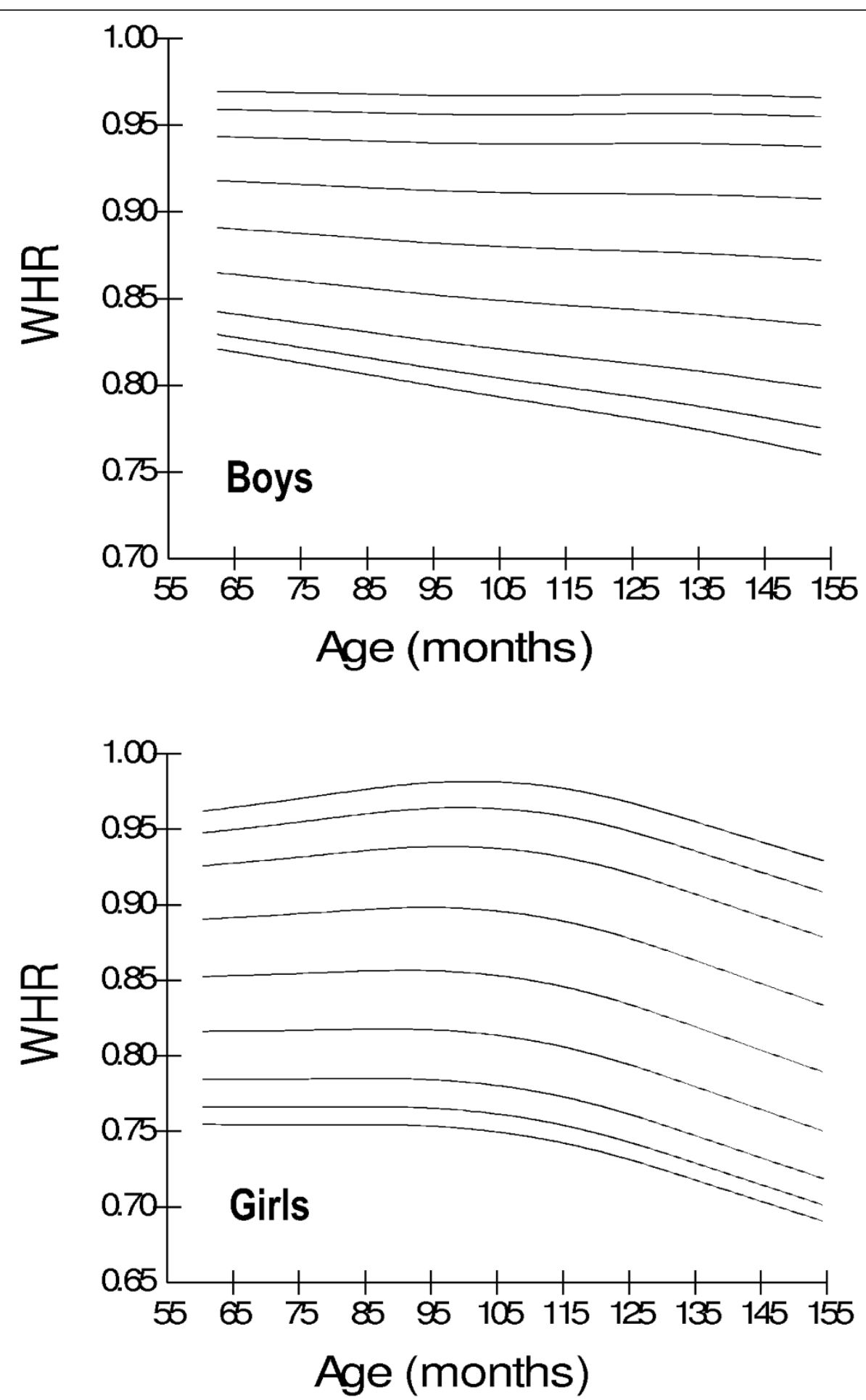

Figure 2 Smoothed waist-hip ratio (WHR) percentile curves for Pakistani primary school boys $(n=997)$ and girls $(n=883)$ aged five to twelve years.

girls with a mean difference of 0.02 . WHtR cut-off of $\geq 0.5$ for defining central obesity corresponded to $85^{\text {th }} \mathrm{WHtR}$ percentile irrespective of age and gender with a mean difference of $<0.01$ [Table 5].
Twelve percent children (95\% CI 10.1-13.0) had a WC $\geq 90^{\text {th }}$ percentile and $16.5 \%$ children $(95 \%$ CI $14.7-18.1)$ had a WHtR $\geq 0.5$ while $11 \%$ children (95\% CI 8.9-11.6) had both WC $\geq 90^{\text {th }}$ percentile and WHtR $\geq 0.5$. 
Table 4 Age- and gender-specific smoothed waist-height ratio (WHtR) percentiles for Pakistani primary school children aged five to twelve years (n = 1860)

\begin{tabular}{|c|c|c|c|c|c|c|c|c|c|}
\hline & \multicolumn{9}{|c|}{ Percentiles } \\
\hline & $3^{\text {rd }}$ & $5^{\text {th }}$ & $10^{\text {th }}$ & $25^{\text {th }}$ & $50^{\text {th }}$ & $75^{\text {th }}$ & $90^{\text {th }}$ & $95^{\text {th }}$ & $97^{\text {th }}$ \\
\hline \multicolumn{10}{|l|}{ Boys $(n=977)$} \\
\hline 5 years (61-71 months) & 0.40 & 0.41 & 0.42 & 0.44 & 0.46 & 0.49 & 0.52 & 0.54 & 0.56 \\
\hline 6 years (72-83 months) & 0.40 & 0.40 & 0.41 & 0.43 & 0.46 & 0.49 & 0.52 & 0.54 & 0.56 \\
\hline 7 years (84-95 months) & 0.39 & 0.39 & 0.40 & 0.43 & 0.45 & 0.48 & 0.52 & 0.54 & 0.56 \\
\hline 8 years (96-107 months) & 0.38 & 0.39 & 0.40 & 0.42 & 0.45 & 0.48 & 0.52 & 0.54 & 0.56 \\
\hline 9 years (108-119 months) & 0.37 & 0.38 & 0.39 & 0.42 & 0.45 & 0.48 & 0.52 & 0.54 & 0.56 \\
\hline 10 years (120-131 months) & 0.37 & 0.37 & 0.39 & 0.41 & 0.44 & 0.48 & 0.52 & 0.54 & 0.56 \\
\hline 11 years (132-143 months) & 0.36 & 0.37 & 0.38 & 0.41 & 0.44 & 0.48 & 0.52 & 0.54 & 0.56 \\
\hline 12 years (144-155 months) & 0.35 & 0.36 & 0.38 & 0.40 & 0.44 & 0.48 & 0.52 & 0.54 & 0.56 \\
\hline \multicolumn{10}{|l|}{ Girls $(n=883)$} \\
\hline 5 years (61-71 months) & 0.39 & 0.40 & 0.41 & 0.43 & 0.45 & 0.48 & 0.51 & 0.53 & 0.54 \\
\hline 6 years (72-83 months) & 0.39 & 0.39 & 0.41 & 0.43 & 0.45 & 0.48 & 0.51 & 0.53 & 0.55 \\
\hline 7 years (84-95 months) & 0.38 & 0.39 & 0.40 & 0.43 & 0.45 & 0.48 & 0.52 & 0.54 & 0.56 \\
\hline 8 years (96-107 months) & 0.38 & 0.39 & 0.40 & 0.42 & 0.45 & 0.49 & 0.52 & 0.55 & 0.57 \\
\hline 9 years (108-119 months) & 0.38 & 0.38 & 0.40 & 0.42 & 0.45 & 0.49 & 0.53 & 0.55 & 0.58 \\
\hline 10 years (120-131 months) & 0.37 & 0.38 & 0.39 & 0.41 & 0.44 & 0.48 & 0.53 & 0.56 & 0.58 \\
\hline 11 years (132-143 months) & 0.36 & 0.37 & 0.38 & 0.41 & 0.44 & 0.48 & 0.53 & 0.56 & 0.59 \\
\hline 12 years (144-155 months) & 0.35 & 0.36 & 0.37 & 0.40 & 0.43 & 0.47 & 0.52 & 0.56 & 0.60 \\
\hline
\end{tabular}



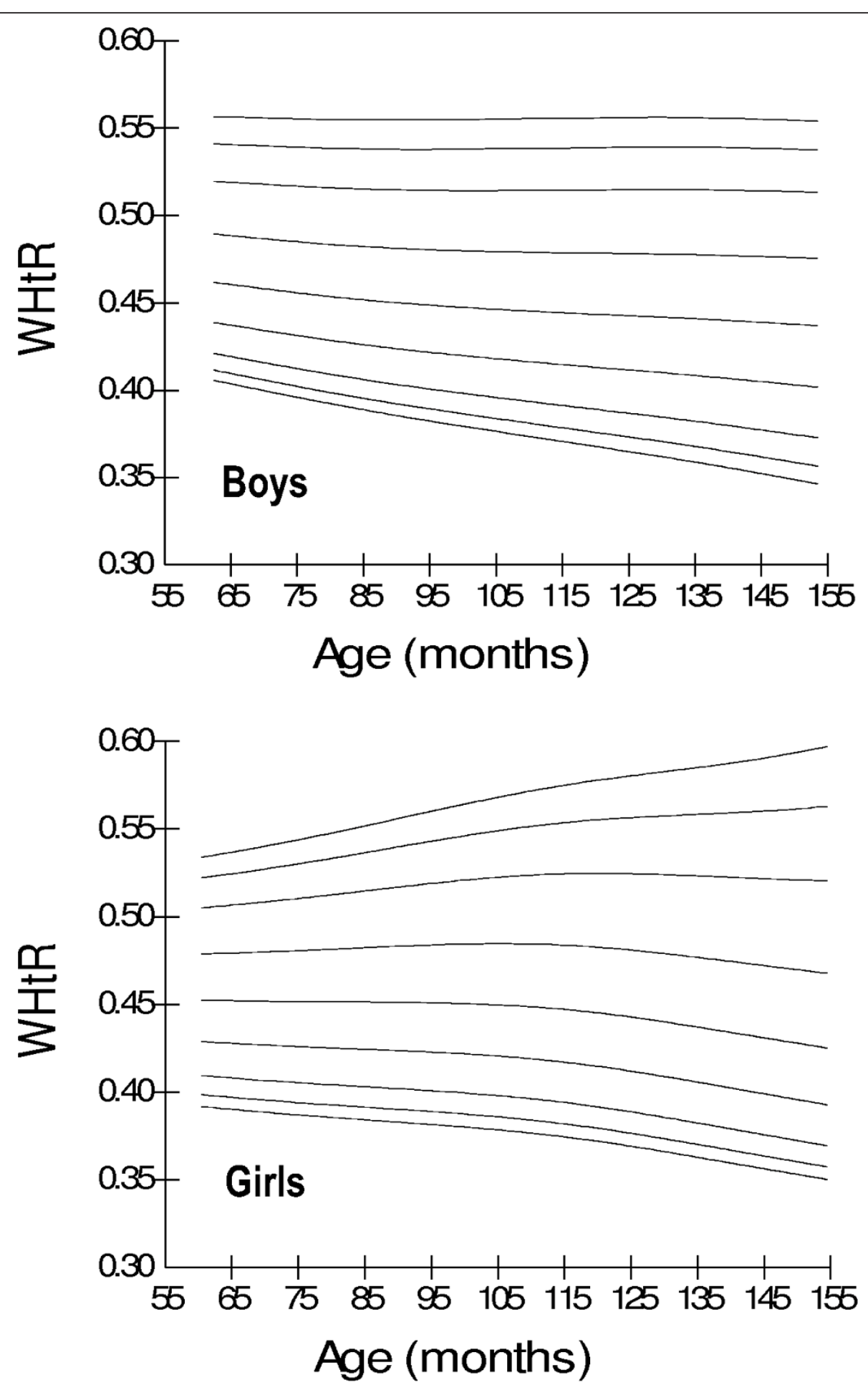

Figure 3 Smoothed waist-height ratio $(\mathrm{WHtR})$ percentile curves for Pakistani primary school boys $(\mathrm{n}=997)$ and girls $(\mathrm{n}=\mathbf{8 8 3})$ aged five to twelve years.

Prevalence of central obesity significantly increased with grade among both boys and girls [Table 6]. Higher grade, urban area with high SES, high-income neighborhood and higher parental education were significantly associated with central obesity (all $\mathrm{P}<0.001$ ) [Table 7]. Multivariate logistic regression analysis was adjusted simultaneously for socio-demographic factors significantly associated with central obesity and controlled for 


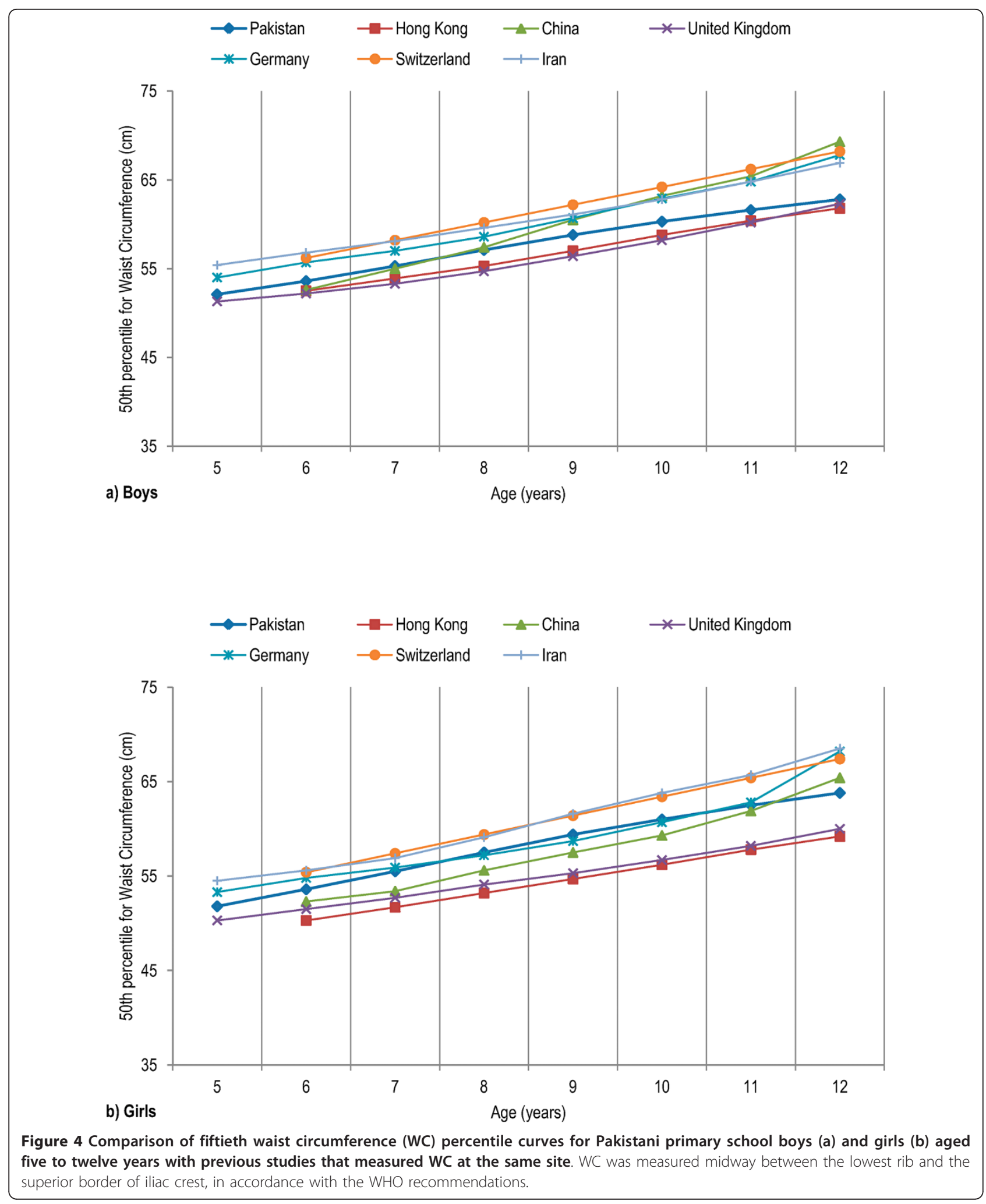

age and gender. Significant independent predictors of central obesity included higher grade (aOR 5.11, 95\% CI 1.76-14.85) and urban area with high SES (aOR 82.34,
95\% CI 15.76-430.31) [Table 8]. Linear regression analyses were adjusted for socio-demographic factors significantly associated with central obesity and controlled for 
Table 5 Age- and gender-specific eighty-fifth percentile values for waist-height ratio (WHtR) corresponding to cut-off value of $\mathbf{0 . 5}$ for defining central obesity among Pakistani primary school children aged five to twelve years $(n=1860)$

\begin{tabular}{lll}
\hline Age & Boys $(\mathbf{n}=\mathbf{9 9 7})$ & Girls $(\mathbf{n}=\mathbf{8 8 3})$ \\
\hline 5 years (61-71 months) & 0.507 & 0.499 \\
6 years (72-83 months) & 0.504 & 0.500 \\
7 years (84-95 months) & 0.502 & 0.504 \\
8 years (96-107 months) & 0.501 & 0.507 \\
9 years (108-119 months) & 0.501 & 0.508 \\
10 years (120-131 months) & 0.501 & 0.507 \\
11 years (132-143 months) & 0.501 & 0.504 \\
12 years (144-155 months) & 0.500 & 0.500 \\
\hline
\end{tabular}

age and gender. Higher grade, urban area with high SES and higher parental education showed a significant independent association with higher WC [Table 9]. Urban area with high SES and higher parental education showed a significant independent association with higher WHtR [Table 10].

\section{Discussion}

First ever age- and gender-specific smoothed percentiles for WC, WHR and WHtR developed from a representative sample of 1860 Pakistani primary school children aged five to twelve years are presented. These charts are based on WC measured midway between the lowest rib and the superior border of iliac crest, in accordance with the WHO recommendations [31].

WC increased with age among both boys and girls, consistent with previous literature [9-24]. There was no gender disparity in WC values, in line with previous findings in children aged five to twelve years [11]. Fiftieth WC percentile curves for Pakistani children were higher as compared to those for Hong Kong and British children, and were lower as compared to those for Iranian, German and Swiss children. WC remains the simplest clinical measure of childhood central obesity. It has been proved a strong predictor of cardiovascular and metabolic disease risk in children [38-47]. It provides a better estimate of visceral adipose tissue than body mass index (BMI) and is significantly more efficient than BMI in predicting insulin resistance, blood pressure, and serum cholesterol and triglyceride levels [38-41]. WHR showed a plateau pattern among boys while remained stable among girls until nine years of age and decreased afterwards, consistent with previous studies [48]. WHR has been used to describe body fat distribution in adults; however, it is influenced by several other body factors and is a poor measure of body fat distribution and risk of related diseases in children $[49,50]$. WHtR was age-independent among both boys and girls, and suggested WHtR cut-off of $\geq 0.5$ for defining central obesity corresponded to $85^{\text {th }} \mathrm{WHtR}$ percentile irrespective of age and gender, in line with previous literature [15,35-37]. Previous studies have strongly correlated WHtR to the risk of cardiovascular and metabolic disease in children, and it has been proposed as an alternative measure for assessing central fatness in children especially for pediatric primary care practice and epidemiological studies, as it is a relatively age-independent measure [8,33,51-56].

Eleven percent of children were centrally obese. Central obesity showed a significantly increasing trend with grade among both boys and girls and higher grade independently predicted the risk of being centrally obese and having higher WC. These findings are consistent with

Table 6 Grade- and Gender-specific prevalence of central obesity among Pakistani primary school children aged five to twelve years $(n=1860)$

\begin{tabular}{|c|c|c|c|c|c|c|}
\hline & \multicolumn{2}{|c|}{$\begin{array}{l}W C \geq 90^{\text {th }} \text { percentile } \\
(\mathrm{n}=213)\end{array}$} & \multicolumn{2}{|l|}{$\begin{array}{l}\text { WHtR } \geq 0.5 \\
(n=304)\end{array}$} & \multicolumn{2}{|c|}{$\begin{array}{l}\text { Centrally Obese }^{a} \\
(n=189)\end{array}$} \\
\hline & n (\%) & P Value & n (\%) & P Value & n (\%) & P Value \\
\hline Boys $(n=997)$ & $103(10.5)$ & & $156(16.2)$ & & $93(9.5)$ & \\
\hline Grade one $(n=233)$ & $19(8.2)$ & 0.054 & $50(21.5)$ & 0.026 & $16(6.9)$ & 0.033 \\
\hline Grade two $(n=187)$ & $13(7.0)$ & & $23(12.3)$ & & $11(5.9)$ & \\
\hline Grade three $(n=183)$ & $18(9.8)$ & & $23(12.6)$ & & $17(9.3)$ & \\
\hline Grade four $(n=184)$ & $26(14.1)$ & & $35(19.0)$ & & 25 (13.6) & \\
\hline Grade five $(n=190)$ & $27(14.2)$ & & $25(13.2)$ & & $24(12.6)$ & \\
\hline Girls $(n=883)$ & $148(16.8)$ & & $110(12.5)$ & & $96(10.9)$ & \\
\hline Grade one $(n=139)$ & $16(11.5)$ & 0.004 & $20(14.4)$ & $<0.001$ & $13(9.4)$ & 0.001 \\
\hline Grade two $(n=185)$ & $11(5.9)$ & & $15(8.1)$ & & $7(3.8)$ & \\
\hline Grade three $(n=189)$ & $31(16.4)$ & & $41(21.7)$ & & $28(14.8)$ & \\
\hline Grade four $(n=188)$ & $33(17.6)$ & & $45(23.9)$ & & $30(16.0)$ & \\
\hline Grade five $(n=182)$ & 19 (10.4) & & $27(14.8)$ & & $18(9.9)$ & \\
\hline
\end{tabular}

${ }^{a} \geq 90^{\text {th }}$ age- and gender-specific waist circumference (WC) percentile and $\geq 0.5$ waist-height ratio (WHtR) 
Table 7 Socio-demographic correlates of central obesity among Pakistani primary school children aged five to twelve years $(n=1860)$

\begin{tabular}{|c|c|c|c|}
\hline \multirow[b]{2}{*}{ Characteristics } & \multirow{2}{*}{$\begin{array}{l}\text { Total Sample }(n=1860) \\
\mathrm{n}(\%)\end{array}$} & \multicolumn{2}{|c|}{ Centrally Obese $^{a}(n=189)$} \\
\hline & & n (\%) & P Value \\
\hline \multicolumn{4}{|l|}{ Gender } \\
\hline Boys & $977(52.5)$ & $93(9.5)$ & 0.335 \\
\hline Girls & $883(47.5)$ & $96(10.9)$ & \\
\hline \multicolumn{4}{|l|}{ Grade } \\
\hline One & $372(20.0)$ & $29(7.8)$ & $<0.001$ \\
\hline Two & $372(20.0)$ & $18(4.8)$ & \\
\hline Three & $372(20.0)$ & $45(12.1)$ & \\
\hline Four & $372(20.0)$ & $55(14.8)$ & \\
\hline Five & $372(20.0)$ & $42(11.3)$ & \\
\hline \multicolumn{4}{|l|}{ Area and socioeconomic status (SES) } \\
\hline Urban with high SES & $465(25.0)$ & $101(21.7)$ & $<0.001$ \\
\hline Urban with middle SES & $465(25.0)$ & $58(12.5)$ & \\
\hline Urban with low SES & $465(25.0)$ & $28(6.0)$ & \\
\hline Rural (low/disadvantaged SES) & $465(25.0)$ & $2(0.4)$ & \\
\hline \multicolumn{4}{|l|}{ Neighborhood income } \\
\hline Low & $651(35.0)$ & $27(4.1)$ & $<0.001$ \\
\hline Middle & $910(48.9)$ & $97(10.7)$ & \\
\hline High & $299(16.1)$ & $65(21.7)$ & \\
\hline \multicolumn{4}{|l|}{ Parental education } \\
\hline Illiterate & $366(19.7)$ & $6(1.6)$ & $<0.001$ \\
\hline High school & $496(26.7)$ & $25(5.0)$ & \\
\hline College & $531(28.5)$ & $71(13.4)$ & \\
\hline Higher education & $467(25.1)$ & 87 (18.6) & \\
\hline
\end{tabular}

${ }^{a} \geq 90^{\text {th }}$ age- and gender-specific waist circumference (WC) percentile and $\geq 0.5$ waist-height ratio (WHtR)

Table 8 Logistic regression analysis of socio-demographic factors associated with central obesity among Pakistani primary school children aged five to twelve years $(n=1860)^{a, b}$

\begin{tabular}{|c|c|c|c|c|}
\hline Characteristics & $\begin{array}{l}\text { Crude OR } \\
(95 \% \mathrm{Cl}) \\
\end{array}$ & P Value & $\begin{array}{l}\text { Adjusted OR } \\
(95 \% \mathrm{Cl})\end{array}$ & P Value \\
\hline \multicolumn{5}{|l|}{ Grade } \\
\hline One & Reference & - & Reference & - \\
\hline Two & $0.70(0.37-1.31)$ & 0.259 & $0.80(0.41-1.56)$ & 0.515 \\
\hline Three & $2.29(1.24-4.22)$ & 0.008 & $3.15(1.54-6.45)$ & 0.002 \\
\hline Four & $3.36(1.66-6.80)$ & 0.001 & $5.47(2.25-13.31)$ & $<0.001$ \\
\hline Five & $2.84(1.23-6.54)$ & 0.014 & $5.11(1.76-14.85)$ & 0.003 \\
\hline \multicolumn{5}{|l|}{ Area and socioeconomic status (SES) } \\
\hline Urban with high SES & $64.24(15.74-262.13)$ & $<0.001$ & $82.34(15.76-430.31)$ & $<0.001$ \\
\hline Urban with middle SES & $32.99(8.01-135.92)$ & $<0.001$ & $35.67(6.95-183.18)$ & $<0.001$ \\
\hline Urban with low SES & $14.83(3.51-62.64)$ & $<0.001$ & $13.67(2.86-65.40)$ & 0.001 \\
\hline Rural (low/disadvantaged SES) & Reference & - & Reference & - \\
\hline \multicolumn{5}{|l|}{ Neighborhood income } \\
\hline Low & $0.16(0.10-0.25)$ & $<0.001$ & $1.50(0.77-2.93)$ & 0.239 \\
\hline Middle & $0.43(0.30-0.61)$ & $<0.001$ & $0.77(0.51-1.17)$ & 0.220 \\
\hline High & Reference & - & Reference & - \\
\hline \multicolumn{5}{|l|}{ Parental education } \\
\hline Illiterate & Reference & - & Reference & - \\
\hline High school & $3.37(1.36-8.31)$ & 0.008 & $0.73(0.26-2.03)$ & 0.545 \\
\hline College & $9.93(4.25-23.20)$ & $<0.001$ & $1.04(0.37-2.91)$ & 0.938 \\
\hline Higher education & $14.13(6.09-32.77)$ & $<0.001$ & $1.32(0.47-3.73)$ & 0.599 \\
\hline
\end{tabular}

${ }^{a}$ The model is controlled for age and gender

${ }^{b} \geq 90^{\text {th }}$ age- and gender-specific waist circumference (WC) percentile and $\geq 0.5$ waist-height ratio (WHtR) 
Table 9 Linear regression analysis of socio-demographic factors with waist circumference (WC) among Pakistani primary school children aged five to twelve years $(n=1860)^{a}$, b

\begin{tabular}{llll}
\hline Characteristics & $\begin{array}{l}\text { Regression coefficient } \\
\mathbf{9 5 \%} \mathbf{C l})\end{array}$ & Standard error & P value \\
\hline Grade & $1.099(0.686$ to 1.511$)$ & 0.210 & $<0.001$ \\
Area and SES & $-1.896(-2.360$ to -1.431$)$ & 0.237 & $<0.001$ \\
Higher neighborhood income & $-0.049(-0.690$ to 0.591$)$ & 0.326 & 0.880 \\
Higher parental education & $0.428(0.018$ to 0.839$)$ & 0.209 & 0.041 \\
\hline
\end{tabular}

${ }^{\mathrm{a}}$ The model is controlled for age and gender

${ }^{\mathrm{b}} \mathrm{R}^{2}$ for model $=0.280$

${ }^{\mathrm{C}}$ Area and SES strata: 1. Urban with high SES, 2. Urban with middle SES, 3. Urban with low SES, and 4. Rural with low/disadvantaged SES

Table 10 Linear regression analysis of socio-demographic factors with waist-height ratio (WHtR) among Pakistani primary school children aged five to twelve years $(n=1860)^{a, b}$

\begin{tabular}{|c|c|c|c|}
\hline Characteristics & $\begin{array}{l}\text { Regression coefficient } \\
(95 \% \mathrm{CI})\end{array}$ & Standard error & $P$ value \\
\hline Grade & $-0.001(-0.004$ to 0.002$)$ & 0.001 & 0.428 \\
\hline Area and SES ${ }^{c}$ & $-0.010(-0.013$ to -0.006$)$ & 0.002 & $<0.001$ \\
\hline Higher neighborhood income & $-0.004(-0.009$ to 0.001$)$ & 0.002 & 0.056 \\
\hline Higher parental education & 0.004 (0.001 to 0.007$)$ & 0.001 & 0.012 \\
\hline
\end{tabular}

${ }^{\mathrm{a}}$ The model is controlled for age and gender

${ }^{\mathrm{b}} \mathrm{R}^{2}$ for model $=0.068$

${ }^{\mathrm{C}}$ Area and SES strata: 1. Urban with high SES, 2. Urban with middle SES, 3. Urban with low SES, and 4. Rural with low/disadvantaged SES

previous studies reporting higher obesity prevalence in older children [57-59]. Urban area with high SES, highincome neighborhood and higher parental education significantly correlated with central obesity. Living in urban area with high SES was a strong independent predictor of being at risk of central obesity. Urban area with high SES and higher parental education were independently associated with higher WC and higher WHtR. Increasing trend in childhood obesity with urbanization had been reported in both the developing and the developed countries [57,60-62]. Association of childhood obesity with high SES and higher parental education had been observed in the developing countries [3,63-68]. However, studies in the developed countries had shown inverse association of SES, neighborhood income level and parental education with obesity [61,69-73]. Different sociocultural circumstances in the developing countries undergoing nutrition transition explain the contradiction. Association between socioeconomic factors and overnutrition vary in societies at different stages of nutrition transition. Obesity is positively associated with socioeconomic factors in Asia but not in Latin America [74]. Changes in lifestyle with urbanization including reduced physical activity, increased sedentary living and unhealthy diets, reinforced by many of the cultural changes associated with globalization, are the probable underlying causes $[60,75,76]$. Efforts to stop childhood obesity should be made on all fronts and targeted interventions, tailored to local circumstances and involving communities, should begin early in life [77].
Variability in the data ascertainment may have introduced an error into the estimates; however, we do not anticipate large or systematic differences. The effects of puberty on anthropometric indices were not explored in the present study; however, future studies are suggested in this regard. Cross-sectional nature of the study should be considered when interpreting the findings reported and future longitudinal studies are warranted to establish the temporal nature and causality of these associations. These findings can be generalized to South Asian primary school children that share the same genetic and environmental factors with the sample.

\section{Conclusions}

Comprehensive worldwide reference values are needed to establish an internationally accepted age-, gender-, and ethnicity-specific definition of central obesity. The present study is the first one to report anthropometric indices predictive of central obesity for Pakistani school-aged children. Eleven percent children were centrally obese and strong predictors included higher grade, urban area with high SES and higher parental education. These findings support the need for developing a National strategy for childhood obesity and implementing targeted interventions, prioritizing the higher social class and involving communities.

\section{Acknowledgements}

We dedicate this work to the loving memories of Ubeera Shahid (1987-2010, Allama lqbal Medical College Class of 2011) who left us in an unfortunate motorway car accident. We are grateful to Professor Arif M Siddiqui, MBBS, 
MRCP, FRCP (Eng, Ed \& Glasg), Usman Khurshid, MBBS, Komal Mushtaq MBBS-IV, Mahar M Shafique, MBBS, Mohsin ZS Ullah, MBBS, M Rizwan, MBBS, Haris S Sheikh, MBBS-III and other Research Associates with us at the Ubeera Memorial Research Society of Allama Iqbal Medical College, Lahore, Pakistan. We would like to thank Dina Kakar, MD, Andrea Lyman, MD, MSc, MS and Douglas Proops MD, MPH. We are indebted to children, parents and staff at the sampled schools. We acknowledge partial financial support from Allama lqbal Medical College and technical support from the Punjab Departments of Health and Education, Lahore, Pakistan.

\section{Author details}

${ }^{1}$ Ubeera Memorial Research Society, Allama Iqbal Medical College, Lahore 54000, Pakistan. ${ }^{2}$ District Health Office Nankana Sahib, Punjab Department of Health, Nankana Sahib 39100, Pakistan.

\section{Authors' contributions}

MUM, principal investigator, conceived and implemented the study, analyzed and interpreted the data, prepared the manuscript and supervised the entire project. SG and HMA contributed to the study analysis, interpretation and manuscript preparation. US contributed to the study conception, implementation and analysis. MAS and JA oversaw the study conception, implementation and manuscript preparation. All authors read and approved the final manuscript.

\section{Competing interests}

The authors declare that they have no competing interests.

Received: 26 July 2011 Accepted: 21 November 2011

Published: 21 November 2011

\section{References}

1. Dietz WH, Robinson TN: Overweight children and adolescents. N Engl J Med 2005, 352:2100-09.

2. Han JC, Lawlor DA, Kimm SYS: Childhood obesity. Lancet 2010, 375:1737-48.

3. Mushtaq MU, Gull S, Abdullah HM, Shahid U, Shad MA, Akram J: Prevalence and socioeconomic correlates of overweight and obesity among Pakistani primary school children. BMC Public Health 2011, 11(1):724.

4. Freedman DS, Dietz WH, Srinivasan SR, Berenson GS: The relation of overweight to cardiovascular risk factors among children and adolescents: the Bogalusa Heart Study. Pediatr 1999, 103:1175-82.

5. Kahn HS, Valdez R: Metabolic risks identified by the combination of enlarged waist and elevated triacylglycerol concentration. Am J Clin Nutr 2003, 78:928-934.

6. Katzmarzyk PT, Srinivasan SR, Chen W, Malina RM, Bouchard C, Berenson GS: Body mass index, waist circumference, and clustering of cardiovascular disease risk factors in a biracial sample of children and adolescents. Pediatrics 2004, 114(2):e198-205

7. Gower BA, Nagy TR, Goran MI: Visceral fat, insulin sensitivity, and lipids in prepubertal children. Diabetes 1999, 48:1515-21.

8. Kelishadi R, Gheiratmand R, Ardalan G, et al: Association of anthropometric indices with cardiovascular disease risk factors among children and adolescents: CASPIAN Study. Int J Cardiol 2007, 117:340-48.

9. Fernandez JR, Redden DT, Pietrobelli A, Allison DB: Waist circumference percentiles in nationally representative samples of African-American, European-American, and Mexican-American children and adolescents. J Pediatr 2004, 145:439-44.

10. Eisenmann JC: Waist circumference percentiles for 7- to 15-year-old Australian children. Acta Paediatrica 2005, 94:1182-85.

11. McCarthy HD, Jarrett KV, Crawley HF: The development of waist circumference percentiles in British children aged 5.0-16.9 y. Eur J Clin Nutr 2001, 55:902-07.

12. Moreno LA, Fleta J, Mur L, Rodriquez G, Sarria A, Bueno M: Waist circumference values in Spanish children-gender related differences. Eur J Clin Nutr 1999, 53:429-33.

13. Savva SC, Kourides Y, Tornaritis M, Epiphaniou-Savva M, Tafouna P, Kafatos A: Reference growth curves for cypriot children 6 to 17 years of age. Obes Res 2001, 9:754-62

14. Fredriks AM, van Buuren S, Fekkes M, Verloove-Vanhorick SP, Wit JM: Are age references for waist circumference, hip circumference and waist-hip ratio in Dutch children useful in clinical practice? Eur J Pediatr 2005, 164:216-22

15. Schwandt $P$, Kelishadi $R$, Haas GM: First reference curves of waist circumference for German children in comparison to international values: the PEP Family Heart Study. World J Pediatr 2008, 4(4):259-66.

16. Aeberli I, Gut-Knabenhans M, Kusche-Ammann RS, Molinari L, Zimmermann MB: Waist circumference and waist-to-height ratio percentiles in a nationally representative sample of 6-13 year old children in Switzerland. Swiss Med Wkly 2011, 141:W13227.

17. Brannsether $B$, Roelants $M$, Bjerknes $R$, Júlíusson P: Waist circumference and waist-to-height ratio in Norwegian children 4-18 years of age: Reference values and cut-off levels. Acta Paediatr 2011.

18. Nawarycz LO, Krzyzaniak A, Stawińska-Witoszyńska B, et al: Percentile distributions of waist circumference for 7-19-year-old Polish children and adolescents. Obes Rev 2010, 11(4):281-8.

19. Ortega FB, Ruiz JR, Vicente-Rodríguez G, Sjöström M: Central adiposity in 9- and 15-year-old Swedish children from the European Youth Heart Study. Int J Pediatr Obes 2008, 3(4):212-6.

20. Sung RYT, So H, Choi K, et al: Waist circumference and waist-to-height ratio of Hong Kong Chinese children. BMC Public Health 2008, 8:324.

21. Kelishadi R, Gouya MM, Ardalan G, et al: First reference curves of waist and hip circumferences in an Asian population of youths: CASPIAN study. J Trop Pediatr 2007, 53:158-164.

22. Hatipoglu N, Ozturk A, Mazicioglu MM, Kurtoglu S, Seyhan S, Lokoglu F: Waist circumference percentiles for 7- to 17-year-old Turkish children and adolescents. Eur J Pediatr 2008, 167(4):383-9.

23. Jackson RT, Al Hamad N, Prakash P, Al Somaie M: Waist circumference percentiles for Kuwaiti children and adolescents. Public Health Nutr 2011, 14(1):70-6.

24. Poh BK, Jannah AN, Chong LK, Ruzita AT, Ismail MN, McCarthy D: Waist circumference percentile curves for Malaysian children and adolescents aged 6.0-16.9 years. Int J Pediatr Obes 2011, 6(3-4):229-35.

25. Novotny R, Daida YG, Grove JS, Le Marchand L, Vijayadeva V: Asian adolescents have a higher trunk:peripheral fat ratio than Whites. J Nutr 2006, 136:642-647

26. He Q, Horlick M, Thornton J, Wang J, Pierson RN Jr, Heshka S, Gallagher D: Sex and race differences in fat distribution among Asian, AfricanAmerican, and Caucasian prepubertal children. J Clin Endocrinol Metab 2002, 87:2164-2170.

27. Khadilkar W, Khadilkar AV, Borade AB, Chiplonkar SA: Body Mass Index Cut-offs for Screening for Childhood Overweight and Obesity in Indian Children. Indian Pediatr 2011

28. Razak F, Anand SS, Shannon H, et al: Defining obesity cut points in a multiethnic population. Circulation 2007, 115:2111-2118.

29. Bhargava SK, Sachdev HS, Fall CH, et al: Relation of serial changes in childhood body-mass index to impaired glucose tolerance in young adulthood. N Engl J Med 2004, 350:865-875.

30. World Health Organization (WHO): Waist circumference and waist-hip ratio: report of a WHO expert consultation. Geneva, 8-11 December 2008. Geneva, Switzerland: WHO; 2011.

31. World Health Organization (WHO): WHO STEPwise approach to surveillance (STEPS). Geneva, Switzerland: WHO; 2008.

32. Cole TJ, Green PJ: Smoothing reference centile curves: the LMS method and penalized likelihood. Stat Med 1992, 11:1305-19.

33. Flores-Huerta $S$, Klünder-Klünder M, Reyes de la Cruz L, Santos JI: Increase in body mass index and waist circumference is associated with high blood pressure in children and adolescents in Mexico city. Arch Med Res 2009, 40(3):208-15.

34. Lee JM, Davis MM, Woolford SJ, Gurney JG: Waist circumference percentile thresholds for identifying adolescents with insulin resistance in clinical practice. Pediatr Diabetes 2009, 10(5):336-42.

35. Nambiar S, Hughes I, Davies PS: Developing waist-to-height ratio cut-offs to define overweight and obesity in children and adolescents. Public Health Nutr 2010, 13(10):1566-74.

36. Ashwell M, Lejeune S, McPherson K: Ratio of waist circumference to height may be better indicator of need for weight management. BMJ 1996, 312:377.

37. Ashwell M: Waist to height ratio and the AshwellR shape chart could predict the health risks of obesity in adults and children in all ethnic groups. Nutrition Food Science 2005, 35:359-64

38. Savva SC, Tornaritis M, Savva ME, et al: Waist circumference and waist-toheight ratio are better predictors of cardiovascular disease risk factors in 
children than body mass index. Int J Obes Relat Metab Disord 2000, 24:1453-58.

39. Lee S, Bacha F, Gungor N, Arslanian SA: Waist circumference is an independent predictor of insulin resistance in black and white youths. J Pediatr 2006, 148:188-94.

40. Maffeis C, Pietrobelli A, Grezzani A, Provera S, Tato L: Waist circumference and cardiovascular risk factors in prepubertal children. Obes Res 2001, 9:179-87.

41. Janssen I, Katzmarzyk PT, Ross R: Waist circumference and not body mass index explains obesity-related health risk. Am J Clin Nutr 2004, 79:379-84.

42. Moreno LA, Pineda I, Rodriguez G, Fleta J, Sarria A, Bueno M: Waist circumference for the screening of the metabolic syndrome in children. Acta Paediatrica 2002, 91:1307-12.

43. Esmaillzadeh A, Mirmiran P, Azizi F: Clustering of metabolic abnormalities in adolescents with the hypertriglyceridemic waist phenotype. Am J Clin Nutr 2006, 83:36-46.

44. Hirschler V, Aranda C, Calcagno ML, Maccalini G, Jadzinsky M: Can waist circumference identify children with the metabolic syndrome? Arch Pediatr Adolesc Med 2005, 159:740-44.

45. Rodríguez-Rodríguez E, Palmeros-Exsome C, López-Sobaler AM, Ortega RM: Preliminary data on the association between waist circumference and insulin resistance in children without a previous diagnosis. Eur J Pediatr 2011, 170(1):35-43

46. Sung RY, Yu CC, Choi KC, et al: Waist circumference and body mass index in Chinese children: cutoff values for predicting cardiovascular risk factors. Int J Obesity 2007, 31:550-58.

47. Hujová Z, Alberty $R$, Ahlers I, et al: Cardiovascular risk predictors in central Slovakian Roma children and adolescents: regional differences. Cent Eur J Public Health 2010, 18(3):139-44.

48. Haas GM, Liepold E, Schwandt P: Percentile curves for fat patterning in German adolescents. World J Pediatr 2011, 7(1):16-23.

49. Moreno LA, Fleta J, Mur L, Feja C, Sarria A, Bueno M: Indices of body fat distribution in Spanish children aged 4.0 to 14.9 years. Journal of Pediatric Gastroenterology \& Nutrition 1997, 25(2):175-81

50. Liu A, Hills AP, Hu X, et al: Waist circumference cut-off values for the prediction of cardiovascular risk factors clustering in Chinese schoolaged children: a cross-sectional study. BMC Public Health 2010, 10:82.

51. Hara M, Saitou E, Iwata F, Okada T, Harada K: Waist-to-height ratio is the best predictor of cardiovascular disease risk factors in Japanese schoolchildren. J Atheroscler Thromb 2002, 9:127-32.

52. Kahn HS, Imperatore G, Cheng YJ: A population-based comparison of BMI percentiles and waist-to-height ratio for identifying cardiovascular risk in youth. J Pediatr 2005, 146:482-88

53. McCarthy HD, Ashwell M: A study of central fatness using waist-to-height ratios in UK children and adolescents over two decades supports the simple message-'keep your waist circumference to less than half your height'. Int J Obesity 2006, 30:988-92.

54. Freedman DS, Kahn HS, Mei Z, et al: Relation of body mass index and waist-to-height ratio to cardiovascular disease risk factors in children and adolescents: the Bogalusa Heart Study. Am J Clin Nutr 2007, 86:33-40.

55. Weili $Y$, He B, Yao H, et al: Waist-to-height ratio is an accurate and easier index for evaluating obesity in children and adolescents. Obesity 2007, 15:748-52

56. Mokha JS, Srinivasan SR, Dasmahapatra P, et al: Utility of waist-to-height ratio in assessing the status of central obesity and related cardiometabolic risk profile among normal weight and overweight/ obese children: the Bogalusa Heart Study. BMC Pediatr 2010, 10:73.

57. Apfelbacher CJ, Loerbroks A, Cairns J, Behrendt H, Ring J, Kramer U: Predictors of overweight and obesity in five to seven-year-old children in Germany: Results from cross-sectional studies. BMC Public Health 2008, 8:171.

58. Jafar TH, Qadri Z, Islam M, Hatcher J, Bhutta ZA, Chaturvedi N: Rise in childhood obesity with persistently high rates of undernutrition among urban school-aged Indo-Asian children. Arch Dis Child 2008, 93(5):373-378.

59. Kinra S, Nelder RP, Lewendon GJ: Deprivation and childhood obesity: a cross sectional study of 20973 children in Plymouth, United Kingdom. J Epidemiol Community Health 2000, 54:456-460.

60. Wang $Y$, Lobstein T: Worldwide trends in childhood overweight and obesity. Int J Pediatr Obes 2006, 1:11-25

61. Wang Y, Beydoun MA: The Obesity Epidemic in the United StatesGender, Age, Socioeconomic, Racial/Ethnic, and Geographic
Characteristics: A Systematic Review and Meta-Regression Analysis. Epidemiol Rev 2007, 29:6-28.

62. Mamalakis G, Kafatos A, Manios Y, Anagnostopoulou A, Apostolaki I: Obesity indices in a cohort of primary school children in Crete: a six year prospective study. Int J Obes 2000, 24:765-771.

63. Hakeem R: Socio-economic differences in height and body mass index of children and adults living in urban areas of Karachi, Pakistan. Eur $J$ Clin Nutr 2001, 55:400-406.

64. Chhatwal J, Verma M, Riar SK: Obesity among pre-adolescent and adolescents of a developing country (India). Asia Pac J Clin Nutr 2004, 13:231-235.

65. Shrewsbury V, Wardle J: Socioeconomic Status and Adiposity in Childhood: A Systematic Review of Cross-sectional Studies 1990-2005. Obesity (Silver Spring) 2008, 16:275-84.

66. Monteiro CA, Conde WL, Lu B, Popkin BM: Obesity and inequities in health in the developing world. Int J Obes 2004, 28:1181-86

67. Wang Y: Cross-national comparison of childhood obesity: the epidemic and the relationship between obesity and socioeconomic status. Int J Epidemiol 2001, 30:1129-1136.

68. Kocaoglu B, Moschonis G, Dimitriou M, et al: Parental educational level and cardiovascular disease risk factors in schoolchildren in large urban areas of Turkey: Directions for public health policy. BMC Public Health 2005, 5:13.

69. Lamerz A, Kuepper-Nybelen J, Wehle C, et al: Social class, parental education, and obesity prevalence in a study of six-year-old children in Germany. Int J Obes (Lond) 2005, 29(4):373-80.

70. Danielzik S, Czerwinski-Mast M, Langnase K, Dilba B, Muller MJ: Parental overweight, socioeconomic status and high birth weight are the major determinants of overweight and obesity in 5-7 y-old children: baseline data of the Kiel Obesity Prevention Study (KOPS). Int J Obes Relat Metab Disord 2004, 28(11):1494-502.

71. Veugelers PJ, Fitzgerald AL: Prevalence of and risk factors for childhood overweight and obesity. CMAJ 2005, 173(6):607-613.

72. Van Lenthe FJ, Mackenbach JP: Neighbourhood deprivation and overweight: the GLOBE study. Int J Obes Relat Metab Disord 2002, 26:234-40.

73. Willms JD, Tremblay MS, Katzmarzyk PT: Geographic and demographic variation in the prevalence of overweight Canadian children. Obes Res 2003, 11:668-73.

74. INCLEN: Body mass index and cardiovascular disease risk factors in seven Asian and five Latin American centers data from the International Clinical Epidemiology Network (INCLEN). Obes Res 1996, 4:221-228.

75. Chopra M, Galbraith S, Darnton-Hill I: A global response to a global problem: the epidemic of overnutrition. Bull World Health Organ 2002, 80:952-958.

76. Popkin BM: An overview on the nutrition transition and its health implications: the Bellagio meeting. Public Health Nutr 2002, 5:93-103.

77. Reilly JJ: Tackling the obesity epidemic: new approaches. Arch Dis Child 2006, 91:724-726.

\section{Pre-publication history}

The pre-publication history for this paper can be accessed here: http://www.biomedcentral.com/1471-2431/11/105/prepub

\section{doi:10.1186/1471-2431-11-105}

Cite this article as: Mushtaq et al:: Waist circumference, waist-hip ratio and waist-height ratio percentiles and central obesity among Pakistani children aged five to twelve years. BMC Pediatrics 2011 11:105. 